\title{
Movilidad en los suburbios dispersos y el Nuevo Urbanismo en los Estados Unidos de América: ¿Importación irreflexiva desde Chile?
}

Constantino Mawromatis P.

\section{Filiación}

Arquitecto de la Universidad de Chile, Bach. of Architecture, University of Miami. Libre ejercicio de la profesión y académico de la F.A.U. de la Universidad de Chile. Actualmente cursa el programa Doctoral Universidad Politécnica de Madrid Universidad de Chile.

\section{Resumen}

El suburbio disperso ha sido el modelo predominante en el crecimiento en los Estados Unidos en el siglo XX. Este modelo ha repercutido dramáticamente en la movilidad de las áreas metropolitanas de los EE.UU., generando una creciente demanda por nuevas infraestructuras y ocasionando un progresivo deterioro en la calidad de vida de sus habitantes. Frente a esta realidad van surgiendo nuevas propuestas que propugnan una planificación neotradicional. En Chile, la suburbanización generada en las últimas dos décadas, controlada en gran medida por fuerzas especulativas, podría verse como una importación irreflexiva de un modelo ajeno, soslayándose las consecuencias negativas que podría tener en el futuro del desarrollo de nuestros asentamientos urbanos.

\section{Palabras Claves}

Suburbio disperso U.S.A, planificación urbana neotradicional, movilidad en áreas metropolitanas.

\begin{abstract}
The disperse suburb has been the most widely used model in the United States during the 20th century. This manner of planning has greatly influenced the mobility within the metropolitan areas in the $U$. S. creating an increasing demand for new infrastructure thus diminishing the standard of living of the inhabitants. Because of this there has been new propositions that suggest to work with neo-traditional planning. In Chile the suburban expansion that has been taking place during the last two decades, mainly due to the posible profits of real estate investments, can be seen as thoughtless attempt to import a foreign model, thus setting aside negative consequences that might affect the future development of our urban settlements.
\end{abstract}

\section{Key words}

Disperse suburbs in the U.S.A., neotraditional urban planning, mobility in metropolitan areas. 


\section{Sumario}

Introducción

1.-El suburbio disperso y consecuencias en la movilidad en los Estados Unidos

2.-La introducción de estos modelos norteamericanos en Chile y la Movilidad: ¿globalización o importación irreflexiva?

Referencias

\section{Introducción}

Con el creciente aumento de la demanda de movilidad durante la segunda mitad del siglo XX en las áreas metropolitanas norteamericanas se genera, a partir de los años 80, un gradual desencanto con el estilo de vida propio de las urbanizaciones residenciales del suburbio disperso en los EEUU.

Los problemas de congestión de tráfico y las consiguientes secuelas económicas, sociales y de salud, son derivados directos e indirectos de su particular modelo de crecimiento.

La construcción de nuevas infraestructuras viales sólo presenta un alivio en una etapa inicial, para luego transformarse en un aliciente para la construcción de nuevas suburbanizaciones y el uso cada vez más masivo y extensivo del automóvil, agravando de esta manera la raíz del problema.

Por otro lado, el encantamiento inicial de la gente frente al desarrollo tecnológico y a las nuevas alternativas de movilidad, sumado a los cambios en las modalidades de trabajo y comunicación, no reemplazan las necesidades de un individuo o grupo humano de sentirse parte de una comunidad, de socializar, y de contactarse con el medio construido y natural. Esto último es, en definitiva, lo que permite formar la identidad individual y colectiva.

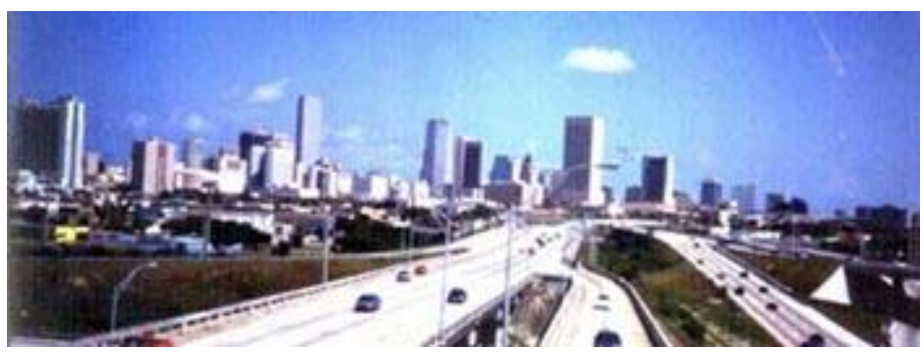

Figura No1: Miami, Florida. Foto de C. Mawromatis P., C.1990. 


\section{1.- El suburbio disperso y consecuencias en la movilidad en los Estados Unidos}

El suburbio como principal modelo de crecimiento se configuró en los EE.UU., esencialmente como una actividad económica, relegándose las consideraciones de diseño urbano a un plano secundario. Ello incidió en la pérdida de protagonismo de los arquitectos y urbanistas en la toma de decisiones referentes a los temas de la ciudad. Se privilegiaron entonces, los factores económicos especulativos, como también consideraciones de orden político en la asignación y división de distritos.

Es así como el paisaje norteamericano está estructurado a partir de la propiedad privada, la cual ha sido determinante en la conformación de las ciudades. La actividad pública por su parte, participó conjunta y paralelamente en el desarrollo y la expansión del territorio (Easterling, 1993).

\section{La aparición del suburbio}

A mediados del siglo XIX apareció el suburbio como una alternativa de crecimiento. El suburbio representó un ideal de vida, ofreciendo seguridad, un ambiente limpio, segregado y cercano a la naturaleza.

En los años 1930, se tomó la decisión política y económica de incrementar y privilegiar el aspecto comercial del desarrollo suburbano residencial, por sobre el compromiso del sector público. En 1934 el Federal Housing Administration (FHA) (1), una agencia gubernamental de fomento y regulación del crecimiento suburbano, elaboró una serie de recomendaciones y proyectos directamente relacionados con la actividad bancaria y comercial que influenciaron de manera fundamental en la materialización del crecimiento del paisaje suburbano. A través de su política de favorecer la estandarización de las soluciones habitacionales acogidas a ésta, se fomentó el desarrollo de grandes urbanizaciones netamente habitacionales. A partir de ese momento histórico el desarrollo de los suburbios se convirtió de hecho, no solamente en una actividad comercial, sino que en una de las principales industrias de los EE.UU., críticamente ligada a la recuperación de la pos-depresión y estrechamente relacionada a la industria automotriz (Easterling, 1993).

\section{La consolidación del suburbio disperso y el posterior desencanto de sus habitantes}

Ya en los años 1950 y por primera vez en su historia, la mayoría de la población de los EE.UU. vivía en suburbios, llegando en el censo de 1980 a constituir aproximadamente el $65 \%$ de la población total (2). Este desarrollo suburbano se ha configurado esencialmente por viviendas unifamiliares aisladas, normadas por el "zoning" como instrumento ordenador y regulador, complicando aún más la estructuración de espacios públicos con forma y destino definidos. Paralelamente se ha promovido la fuerte inversión en infraestructura vial, concentrándose en la creación de autopistas que conectan las distintas zonas de la ciudad y al territorio en general. 


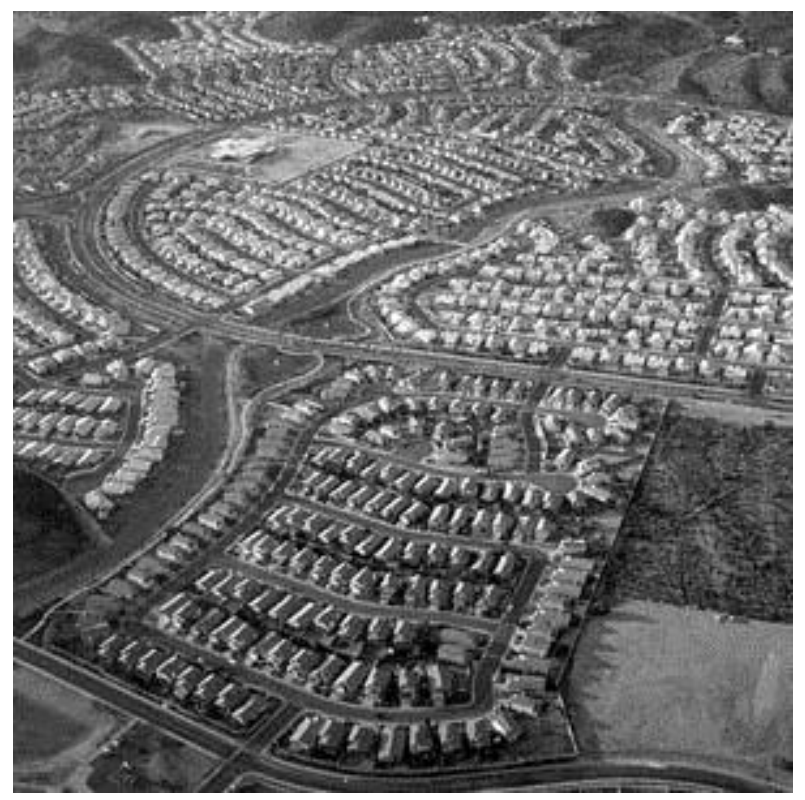

Figura N02: Subdivisión al sur-este de Phoenix, Arizona.

Fuente: Land Lines, January 2002, Newsletter of the Lincoln Institute of Land Policy. Sección de foto original en color, de Alex Mac Lean, Landslides Aerial Photography, Cambridge, Massachusetts.

Reproducción sin fines de lucro, con objetivos estrictamente culturales.

Esto por su parte, ha incentivado la creación de nuevos suburbios residenciales, preferentemente en la periferia de la ciudad, en donde los costos aún hoy se mantienen bajos, con el consiguiente beneficio económico para la inversión privada y el consecuente daño al entorno construido y al natural, deteriorando en última instancia la calidad de vida de los ciudadanos.

Según señala el ingeniero español, Dr. Ing. Julio Pozueta (en 1991-92), las políticas que apuntan hacia la dirección de aumentar la infraestructura vial -opción con la que se afrontó en los 60 y 70 los aumentos de demanda y movilidad- resulta hoy día difícil de realizar en estas áreas, tanto por el encarecimiento de tales obras, que requerirían en muchos casos la remodelación de barrios recientemente construidos y la adquisición de suelos muy costosos, como por las progresivas exigencias de calidad ambiental de los habitantes de las áreas urbanas y suburbanas, que tienden a limitar la expansión de la circulación del automóvil.

Es así como el habitante de las áreas metropolitanas norteamericanas destina un importante porcentaje de su día en las autopistas. El automóvil, que representó en la cultura norteamericana por buena parte del siglo XX la máxima expresión de libertad individual, y que posibilitó el desplazamiento por el territorio a través de la flamante red vial, se ha convertido hoy en el medio obligado para efectuar las tareas diarias tales como el trabajo, educación, recreación, compras, etc. 


\section{REVISTA DE URBANISMO \\ ISSN 0717-5051}

http://revistaurbanismo.vchile.cl

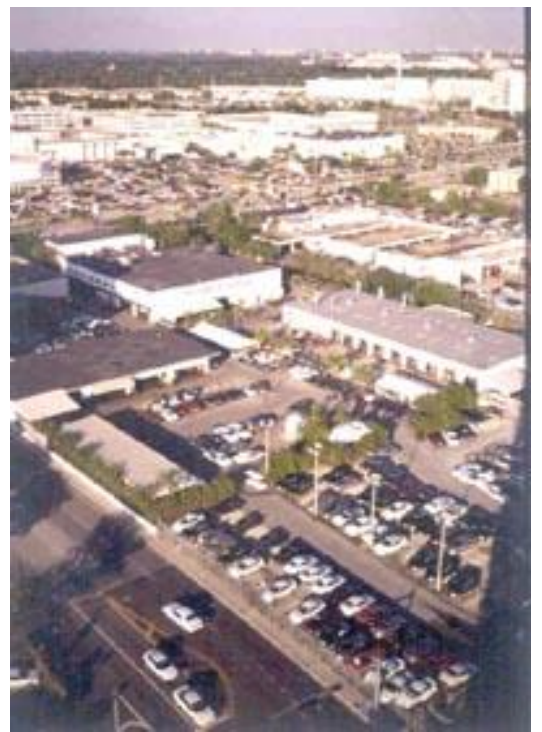

Movilidad en los suburbios dispersos y el Nuevo Urbanismo en los Estados Unidos de América:

¿Importación irreflexiva desde Chile?

Figuras N03 y 4: Miami, Florida.

Fotos de C. Mawromatis P., 1997.

\section{EI Nuevo Urbanismo frente a los desafíos de la movilidad}

Frente a la creciente insatisfacción y frustración que existe en el norteamericano medio, con respecto a su medio ambiente construido del suburbio disperso y a la problemática de movilidad que se contrapone con el ideal de libertad individual, han surgido con gran aceptación de la crítica especializada y de la opinión pública, iniciativas para incorporar el diseño urbano como variable fundamental en los nuevos proyectos de los promotores inmobiliarios. Estas nuevas iniciativas de orientación neotradicional, reintroducen los usos mixtos y otros variados aspectos de los poblados tradicionales de los EE.UU. (Traditional Towns), que refuerzan el sentido de una comunidad compacta y la identidad para con su medio ambiente construido. 


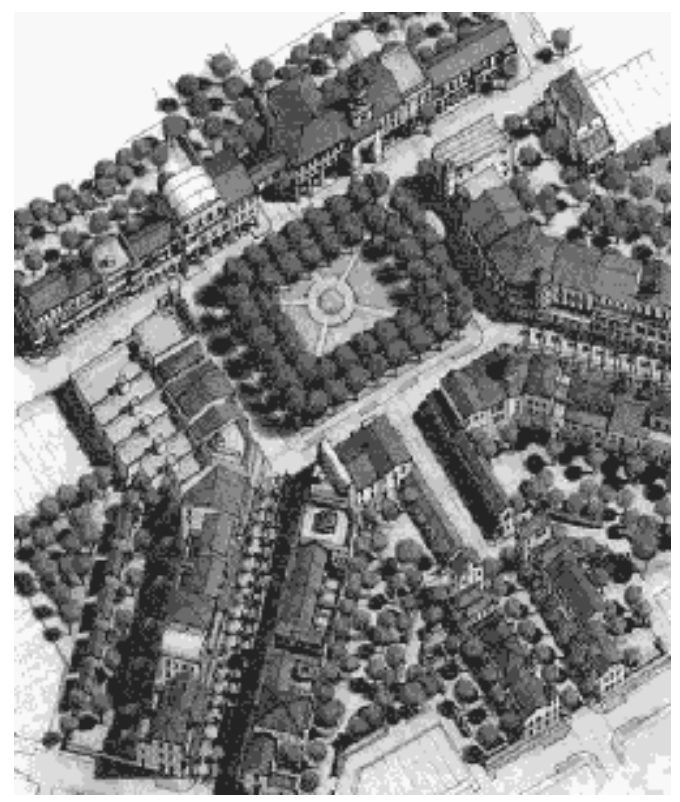

Figura N05: Proyecto "A New Village in the Suburbs", situado en Dade County, Florida 1992, Arqtos. Dover, Kohl \& Partners.

Fuente: KATZ, Peter, The New Urbanism Toward an Architecture of Community, Ed. Mc Graw-Hill, Inc. Dibujo original en color, de Maricé Chael, p. 98. Reproducción sin fines de lucro, con objetivos estrictamente culturales.

\section{EI Nuevo Urbanismo}

Agrupados en un movimiento llamado el "New Urbanism" (Nuevo Urbanismo), un número creciente de arquitectos y urbanistas han adherido a una visión más integral del urbanismo que la mera zonificación funcional y el ordenamiento vial vehicular, rescatando los valores de los poblados tradicionales de norteamérica.

En contraste con los suburbios dispersos, la planificación neotradicional se caracteriza por la incorporación de usos mixtos, densidades mayores, incorporación de transporte público, vías peatonales y ciclovías. Además, estos proyectos presentan una mayor conectividad con las redes viales y con el tejido urbano existente.

Como alternativas a los instrumentos de regulación vigentes en los EE.UU., el "New Urbanism" propone normativas propias como el TND (Traditional Neighborhood Development), de Duany and Plater-Zyberk, y el TOD (Transit Oriented Development), de Peter Calthorpe. 


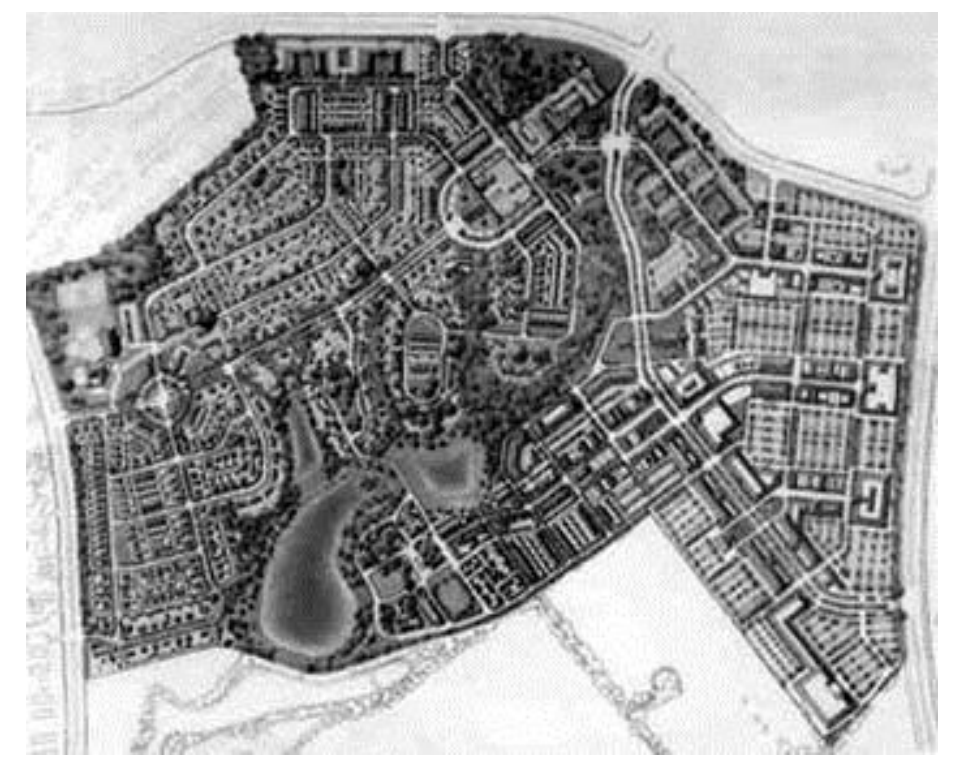

Figura N06: Proyecto "The Town of Kentlands", Gaithersburg, Maryland, 1988, Arqtos. Duany \& Plater-Zyberk;

Fuente: KATZ, Peter, The New Urbanism Toward an Architecture of Community, Ed. Mc Graw-Hill, Inc.

Dibujo original en color, de Maricé Chael, p. 31. Reproducción sin fines de lucro, con objetivos estrictamente culturales.

\section{La planificación neotradicional y la movilidad}

La primera experiencia de planificación neotradicional (TNC - Traditional Neighborhood Communities) en los EE.UU., se materializa en el proyecto de un asentamiento costero en el norte del estado de Florida que surge de un visionario promotor inmobiliario, Robert Davis, el cual encarga el proyecto a la oficina de los entonces noveles arquitectos y diseñadores urbanos Andres Duany y Elizabeth Plater-Zyberk. Estos plantean desarrollar un proyecto urbanístico llamado Seaside (TND) que recoge la estructura y morfología de los poblados tradicionales norteamericanos, incorporando al automóvil como elemento ineludible de la movilidad contemporánea. 


\section{REVISTA DE \\ URB}

http://revistaurbanismo.uchile.cl
Movilidad en los suburbios dispersos y el Nuevo Urbanismo en los Estados Unidos de América:

¿Importación irreflexiva desde Chile?

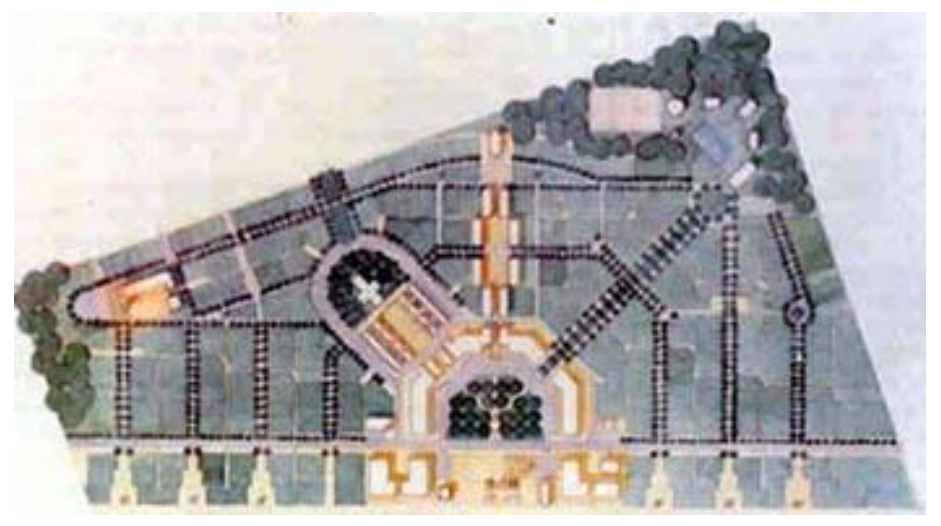

Figura N07: Planta de proyecto "Seaside", Walton County, Florida, 1981, Arqtos. Duany \& Plater-Zyberk;

Fuente: KATZ, Peter, The New Urbanism Toward an Architecture of Community, Ed. Mc Graw-Hill, Inc. Sección de foto original color, de Steven Brooke, p. 3. Reproducción sin fines de lucro, con objetivos estrictamente culturales.

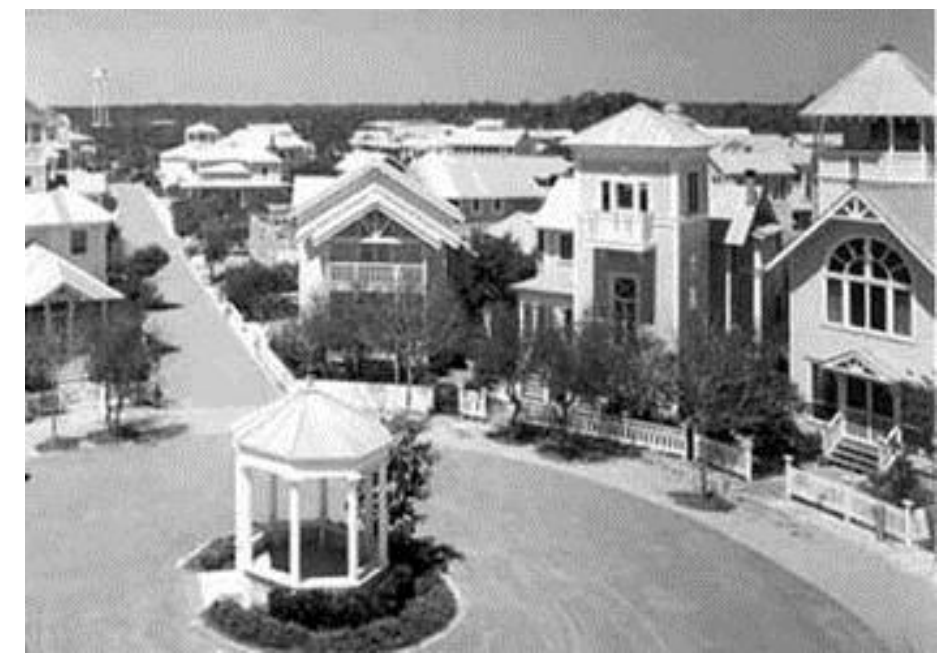

Figura N08: Vista de "Seaside", Walton County, Florida, 1981; Arqtos. Duany \& Plater-Zyberk

Fuente: KATZ, Peter, The New Urbanism Toward an Architecture of Community, Ed. Mc Graw-Hill, Inc. Sección de foto original color, de Steven Brooke, p.13. Reproducción sin fines de lucro, con objetivos estrictamente culturales. 
Además de un fuerte componente morfológico en la configuración de Seaside, se ha puesto especial énfasis en la conformación de espacios públicos, destacando su plaza principal y las circulaciones peatonales exclusivas en circuitos interiores de las manzanas, valorando de este modo la movilidad peatonal local y promoviendo de esta manera una vida más comunitaria y en contacto con la naturaleza.

Seaside y los siguientes proyectos de urbanismo neotradicional norteamericanos, ejemplifican de buena manera como conjugar la movilidad meramente funcional (conectividad del punto A al punto B) con la experiencia sensorial más compleja que significa el hecho de trasladarse de un lugar a otro. Las calles boulevares, paseos, etc., se articulan y se encadenan en secuencias rotuladas por espacios y edificios públicos de significación, de tal manera de orientar, amenizar y caracterizar la función de la movilidad. Ello adquiere vital importancia en el caso de desplazamientos peatonales, ya que si se carece de tales estímulos se opta por modalidades de movilidad alternativas.

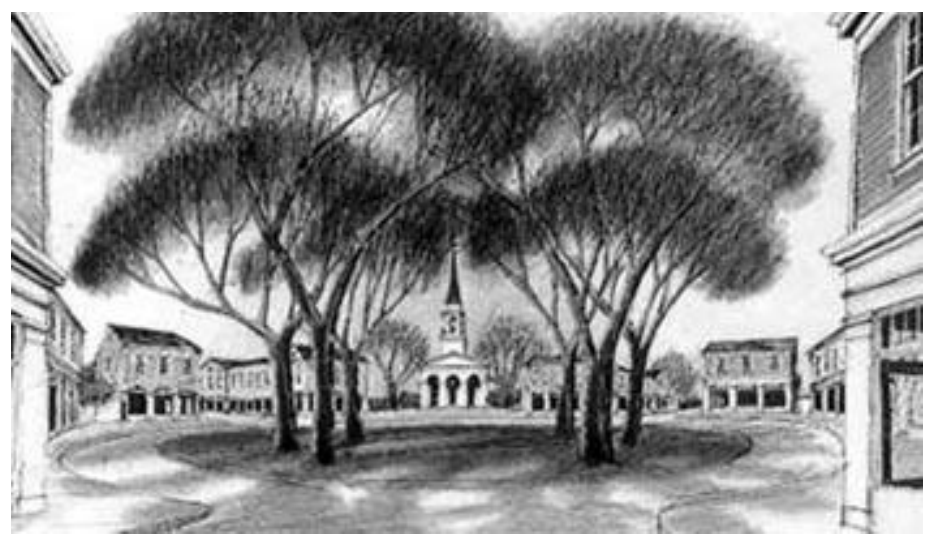

Figura No9. Proyecto "The Village of Mashpee Commons", Cape Cod, Massachusetts, 1986, Arqtos. Duany \& Plater-Zyberk.

Fuente: KATZ, Peter, The New Urbanism Toward an Architecture of Community, Ed. Mc Graw-Hill, Inc. Dibujo de Charles Barret, original color, p. 172. Reproducción sin fines de lucro, con objetivos estrictamente culturales.

Las calles del Nuevo Urbanismo se conciben como parte integral del diseño urbano y se clasifican de acuerdo a sus características de movilidad y a tipologías y jerarquías que corresponden no tan solo a capacidades de tráfico, sino también a su ubicación relativa dentro de la estructura urbana.

Otro aspecto central del Nuevo Urbanismo en relación con la movilidad peatonal, se refiere a privilegiar mayores densidades cercanas al comercio y servicios para así posibilitar que un mayor número de residentes se encuentre a distancia de marcha a pie, de ellos, tal es el caso por ejemplo de Laguna West de Peter Calthorpe. 


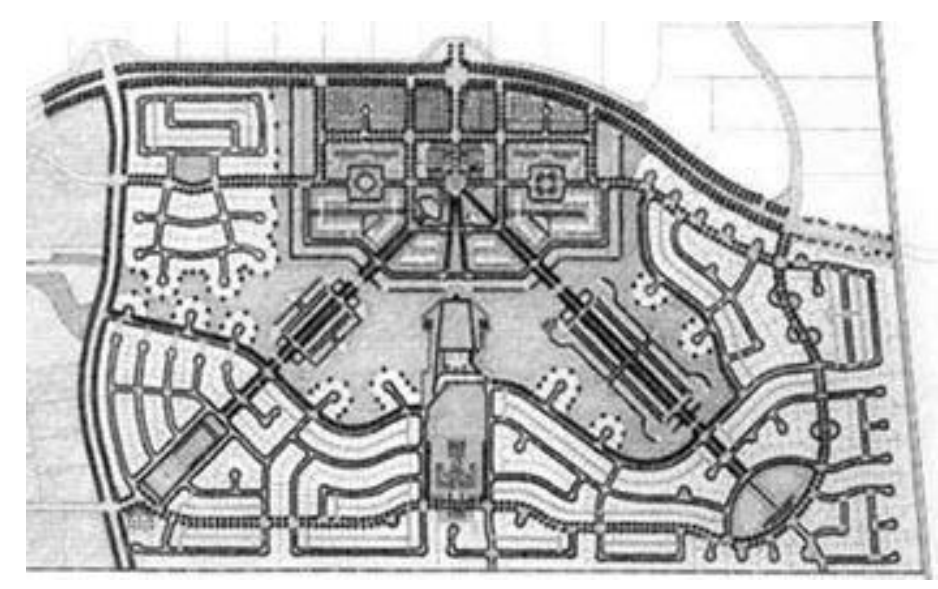

Figura N010: Proyecto "Laguna West", Sacramento County, California, 1990, Arqtos. Calthorpe Associates;

Fuente: KATZ, Peter, The New Urbanism Toward an Architecture of Community, Ed. Mc Graw-Hill, Inc. Dibujo s/d, original color, p. 19. Reproducción sin fines de lucro, con objetivos estrictamente culturales.

\section{EI Transit Oriented Development, de Peter Calthorpe}

Peter Calthorpe (1993) plantea un diseño urbano orientado a la movilidad. El TOD (Transit Oriented Development) conlleva principios de usos de suelos básicos tales como la localización de la vivienda y la tipología y proximidad del comercio minorista.

EI TOD se refiere a directrices que definen una estrategia integrada del crecimiento de ciudades, suburbios y towns. Se intenta con ello definir un nuevo contexto y dirección para el medio ambiente construido.

Para Peter Calthorpe es esencial considerar al peatón como catalizador de la planificación urbana. Ello no significa que el tráfico vehicular pase a un segundo plano, sino que necesariamente el peatón debe ser considerado como variable esencial en la planificación. Con ello, agrega Calthorpe, los suburbios se convertirán en verdaderas ciudades, las urbanizaciones en barrios y las redes o networks en comunidades.

Teniendo en cuenta al peatón como pieza esencial en la estructuración de un barrio, se requiere definir el tamaño óptimo de una comunidad, considerando la relación de las actividades y necesidades del usuario-peatón. El tamaño es una variable primordial para el buen funcionamiento de una comunidad. Este, se determina en gran medida por las distancias a recorrer por un peatón en las diversas actividades diarias que realiza en su entorno. 


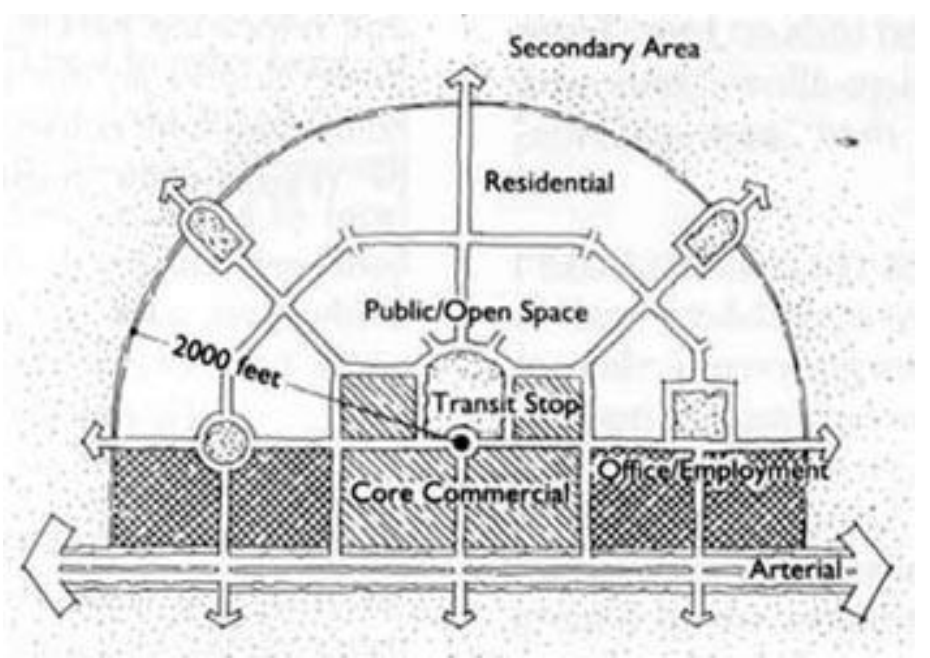

Figura No11: Transit-Oriented Development (T.O.D.), Arqto. Peter Calthorpe. Fuente: CALTHORPE, Peter, 1993. The Next American Metropolis, Ecology, Community, and the American Dream. Princeton Architectural Press, p.56. Reproducción sin fines de lucro, con objetivos estrictamente culturales.

Las distancias físicas a recorrer por un peatón, las que definirían el radio relativo de un poblado o barrio, no debiera exceder los 10 minutos de caminata, lapso considerado por varios autores como el máximo al cual está dispuesta una persona a caminar sin optar preferencialmente por el auto o la locomoción colectiva.

En síntesis los principios del TOD (Transit Oriented Development) son:

- Organizar el crecimiento a un nivel regional, de manera compacta y sustentable en la movilidad.

- Ubicar los usos comerciales, habitacionales, laborales, recreacionales (parques) y cívicos, a distancias de marcha a pié de estaciones de cambio modal (Transit Stops). - Crear redes de calles "amistosas" para con el peatón, y que conecten destinos locales.

- Proveer de una diversidad de viviendas en cuanto a su tipología, densidad y costo. - Preservar hábitats ecológicamente frágiles, y espacios abiertos de gran calidad. - Hacer de los espacios públicos, el foco de orientación de edificios y de las actividades de los barrios.

- Promover la renovación urbana.

Estos principios, tal como los presenta Calthorpe, no pretenden ser novedosos, sino rescatar las metas del urbanismo en su verdadero sentido. 


\section{El Tradicional Neighborhood Development, de Duany And Plater-Zyberk}

Por otro lado, Duany and Plater-Zyberk proponen una normativa alternativa al PDU (Planned Unit Development), el TND o Tradicional Neighborhood Development. Las ordenanzas del TND restablecen la opción de crear nuevas urbanizaciones con la estructura de la ciudad tradicional con el siguiente criterio:

1.- El barrio es limitado en tamaño, con bordes y un centro definido.

2.- Comercio, oficinas, colegios y vivienda para todas las clases sociales se encuentran próximos.

3. Las calles se dimensionan y diseñan para servir equitativamente a las necesidades del automóvil y a las del peatón.

4.- El tamaño y carácter de los edificios están normados para definir espacialmente las calles y plazas.

5.- Plazas y parques se distribuyen y diseñan como lugares de actividad social y recreativa.

6.- Los edificios cívicos se localizan estratégicamente actuando como símbolos de la identidad de la comunidad y como lugar de asamblea para la misma.

Estos criterios persiguen ciertos objetivos sociales:

1.- La organización compacta reduce la necesidad de infraestructura, el uso del automóvil y la contaminación, mientras que facilita el tránsito público.

2.- El amplio espectro de tipologías en lo residencial y trabajo, fomenta la integración de todos los grupos de edades y de clases económicas.

3.- La provisión de espacios públicos confortables permite una mejor interacción entre vecinos ayudando a la seguridad interna.

4.- La provisión de las necesidades del diario vivir dentro del barrio y a distancia peatonal, permite mayor independencia a la población juvenil y a la tercera edad.

5.- Los edificios públicos apropiados están dirigidos a favorecer las iniciativas democráticas y a la evolución equilibrada de una sociedad.

También se establecen tipologías que definen el carácter del espacio público. El objetivo apunta a la seguridad y confort del peatón, como también la expedita circulación del tráfico vehicular. Se especifican claramente la proporción del ancho de la calle respecto a la altura de la edificación; las secciones de las pistas de circulación y 
estacionamiento; la alineación de árboles; y las dimensiones de la vereda. Las variaciones en las tipologías tienen relación tanto en el carácter urbano o rural de la calle como también a aspectos utilitarios. Un cuadro tipológico completo puede incluir lo siguiente: autopistas, avenidas y bulevares para el tráfico regional, vías para tráfico de alta densidad tanto residencial como comercial, vías para tráfico en áreas residenciales de baja densidad, y pasajes locales.

\section{El peatón como protagonista en la movilidad}

Como criterio general, el Nuevo Urbanismo establece también la configuración de predios y viviendas de diversos tamaños, privilegiando la diversidad y apuntando hacia una conformación compacta. Ello se logra incluso con densidades relativamente bajas y viviendas aisladas, privilegiando el espacio público por sobre el jardín privado. De esta manera se logra reducir las distancias de los desplazamientos tales como visitas a servicios, comercio minorista, colegios, etc. y en ciertos casos (TOD) a la estación de cambio de medio más cercana.

Se trata por lo tanto de crear las condiciones para favorecer el desplazamiento peatonal, es decir una ciudad "paseable".

En el caso del TOD, se considera un promedio de diez minutos de marcha a pié, como la distancia entre los lotes residenciales más lejanos y el paradero o estación de cambio de medio, lo cual se traduce en aproximadamente 2000 pies (610 metros) de radio máximo.

Previamente, Leon Krier, establece igualmente, que la distancia máxima que un peatón está dispuesto a recorrer cómodamente sin recurrir a otras alternativas de movilidad, equivale a una marcha a pié de diez minutos, lo cual determina últimamente el radio de una comunidad "paseable".

Duany and Plater-Zyberk por su parte, en su modelo del TND (Traditional Neighborhood Development), configuran una comunidad o barrio que varía en su tamaño desde los 40 acres (16.2 Ha.) a los 200 acres (81 Ha.), teniendo en cuenta no sobrepasar un radio de más de un cuarto de milla $(402 \mathrm{~m}$.). De esta manera se asegura que la distancia entre la mayoría de las viviendas y los parques de barrio se encuentren a tres minutos de marcha a pié, y a cinco minutos de una plaza central (cívica) con servicios y estación de cambio de medio.

Según el Dr. Julio Pozueta, hacer una ciudad "paseable", implica que sus recorridos puedan producirse en condiciones adecuadas, para lo cual es conveniente promover una red de itinerarios peatonales principales, la réplica peatonal a las redes arteriales para vehículos, que articulen el conjunto de las áreas urbanas asegurando las conexiones entre los grandes generadores de desplazamiento.

En general, y al igual que sucede con los ciclistas, los itinerarios peatonales deben cumplir una serie de cualidades, debiendo ser: 
- Funcionales, es decir, que conecten los principales focos de generación - atracción de viajes, sin obligar a rodeos o esperas innecesarias. Especialmente importante, en este sentido, es la conexión peatonal a las estaciones y paradas de transporte público, así como a los centros de empleo, escuelas, comercio, ocio, centros culturales, etc.

-Seguros, tanto con respecto a los vehículos (separación de calzada, cruces preferentes, etc.), como a posibles comportamientos antisociales (itinerarios autovigilados, ausencia de lugares ocultos, iluminación, etc.).

- Confortables, es decir, amplios, bien pavimentados, de pendientes moderadas, poco ruidosos, con zonas de sombra y protección frente a la lluvia, equipados (bancos, teléfonos, etc.).

- Atractivos, bien por atravesar zonas de actividad y animación, bien por las vistas y panoramas que proporcionan, bien por el ritmo y la secuencia de hitos, monumentos, etc.
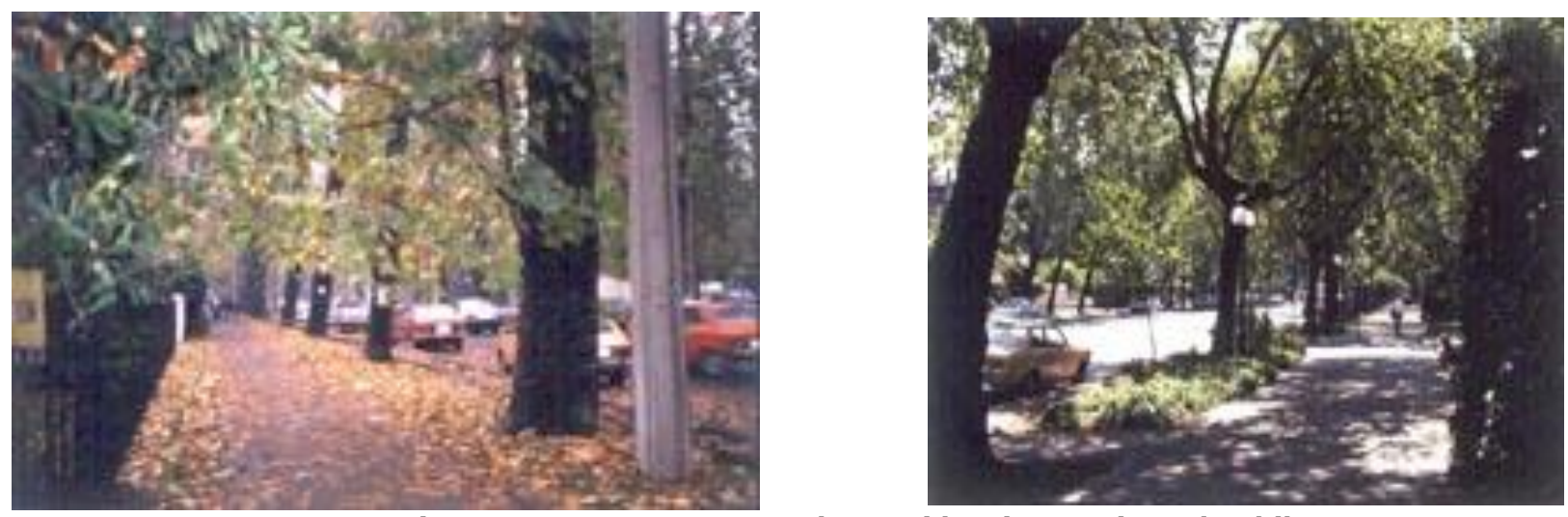

Figura No12 y 13: Comuna de Providencia, Santiago de Chile.

Foto de C. Mawromatis P., 1994. 


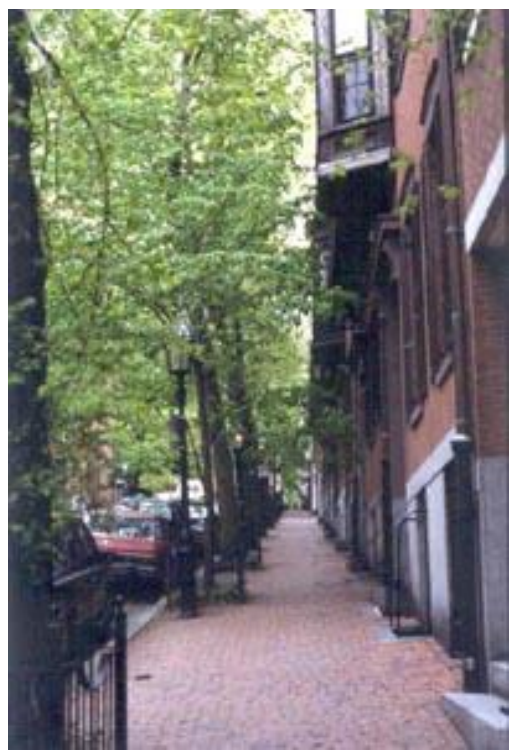

Figura No14: Boston, Massachusetts, EE.UU.

Foto de C. Mawromatis P., 1997.

Más allá de una cuestión puramente del ámbito de la planificación o el diseño urbano, el automóvil como principal protagonista de la movilidad en las áreas metropolitanas es propio de una idiosincrasia y modo de vida que se encuentra profundamente arraigada en la psiquis social e individual del norteamericano medio.

Tal como en las ciudades europeas, en las cuales sus habitantes valoran, respetan e interactúan en sus centros históricos teniendo como marco físico para sus actividades y ritos al espacio urbano contenido y conformado, el norteamericano medio se inserta en una llamada cultura del suburbio, dentro de un esquema de obligada movilidad vehicular para cada actividad que realiza fuera de su hogar. Esta relación íntima entre el auto y el individuo, nos lleva a reflexionar y evaluar hasta qué punto las propuestas del Nuevo Urbanismo, pueden funcionar eficaz y eficientemente como un proyecto global, más allá de experimentos localizados y focalizados en experiencias particulares.

En efecto, el norteamericano medio cambia de vivienda con creciente frecuencia, lo cual muchas veces significa un cambio de localidad, ciudad o estado. Los towns norteamericanos que se presentan como precedentes para el Nuevo Urbanismo, se estructuran a partir de una organización familiar y social muy diferente a la cultura contemporánea en los EE.UU.

Por lo tanto, al rescatar los valores de los llamados "traditional towns", lo que se hace es rememorar la configuración física. Sin embargo, la verdadera esencia de la vida comunitaria, los lazos sociales y el sentido de pertenencia que argumenta el Nuevo Urbanismo como principios estructuradores de una ciudad, no corresponde 
enteramente a la realidad de la sociedad contemporánea ni menos a una tendencia a largo plazo.

Según podemos apreciar en diversas culturas desarrolladas, y en vías de desarrollo, la sociedad tiende más bien a una mayor diversidad del núcleo familiar tradicional. El creciente individualismo y la reducción del número de integrantes de una familia es un fenómeno global en sociedades emergentes. Esto sumado a una creciente movilidad con distancias y frecuencias (trabajo, localidad) cada vez mayores, confirman el evidente desmembramiento de las estructuras familiares y sociales conocidas en sociedades llamadas tradicionales y con ello se introduce una variable de mayor complejidad para la planificación de nuestros ambientes construidos.

Estas variables son fundamentales de incluir en un análisis y evaluación global de los principios del Nuevo Urbanismo para asegurar que estos modelos puedan considerar e incluir la complejidad económica-social, además del creciente dinamismo del urbanismo contemporáneo.

\section{La introducción de estos modelos norteamericanos en Chile y la movilidad: ¿globalización o importación irreflexiva?}

Frente al creciente protagonismo de los EE.UU. en la política y economía internacional, el crecimiento de países en vías de desarrollo como Chile, está sujeto en gran medida a modelos probados y consolidados en norteamérica.

Si bien en los EE.UU. tanto el suburbio disperso, como el urbanismo neotradicional obedecen a raíces históricas y a las aspiraciones de sus gentes, frente a la globalización cultural, nos acercamos cada vez más a muchas de las estructuras sociales propias del modelo norteamericano. Ante este hecho y en el marco de la planificación urbana, podemos anticipar que los modelos norteamericanos seguirán insertándose en nuestra realidad cada vez con más fuerza.

Ello ya se puede apreciar en la modalidad de crecimiento suburbano por sumatoria de predios agrícolas, que se está efectuando hace un par de décadas en la periferia urbana de Santiago, y también en diversos proyectos inmobiliarios -oferta de una segunda vivienda, para recreación- en las costas de Chile central.

No obstante lo anterior, el desafío consiste en adaptar a la realidad local, las nuevas alternativas de regulación del desarrollo y la renovación urbana, que han surgido en la última década en los EE.UU. 

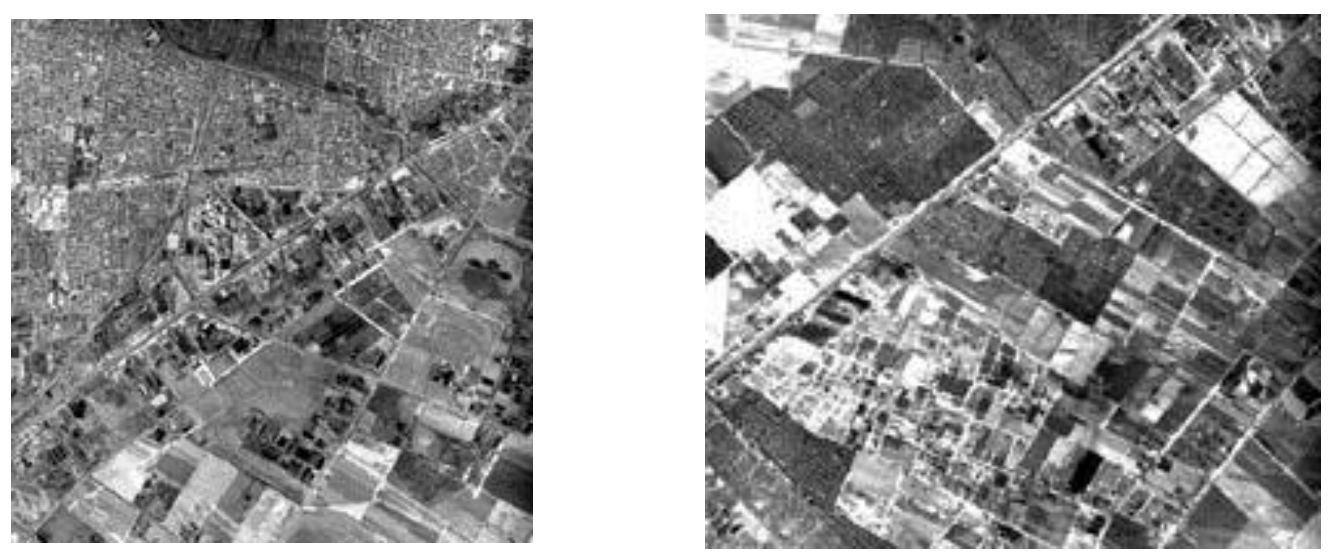

Figuras No15 y 16: Comuna de Maipú, Santiago de Chile. Crecimiento por sumatoria de predios agrícolas. Fuente: Servicio Aerofotogramétrico de la Fuerza Aérea de Chile, 1996

En cuanto al tema de la movilidad, se debe considerar el dinamismo que presenta una sociedad emergente de altos índices de crecimiento en sus áreas metropolitanas. El desarrollo que ha estado experimentando Chile y la progresiva disminución de las tasas de pobreza, incorporará al mercado a un creciente número de familias que podrán tener acceso a diversos bienes, tales como el automóvil. Este solo hecho nos lleva a revisar y analizar la experiencia estadounidense de la primera mitad del siglo $\mathrm{XX}$, la cual fue determinante en la configuración del desarrollo urbanístico de ese país y en la problemática en la movilidad que hoy se puede apreciar en la mayoría de las urbes norteamericanas.

\section{El caso de Santiago de Chile y la política urbana detonante del suburbio disperso}

Si nos trasladamos a la realidad chilena, en concreto a la situación de Santiago, tendremos que tomar en cuenta los diversos factores que pueden incidir en las limitaciones y potencialidades que tienen o pudiesen tener estas experiencias de modelos importados, muchas veces irreflexivamente, desencadenando las problemáticas ya conocidas en los países desarrollados, en este caso EE.UU., y ocasionando otras posibles consecuencias que derivan de la complejidad de un tejido urbano y social de raíz distinta y diversa frente a la cultura norteamericana.

Ello ya se ha reflejado en ciertas políticas de desarrollo urbano, que siguiendo el modelo de crecimiento del suburbio disperso norteamericano, introdujeron una mecánica de crecimiento en la Región Metropolitana de Santiago que ha generado una serie de consecuencias de gran impacto en la movilidad. 


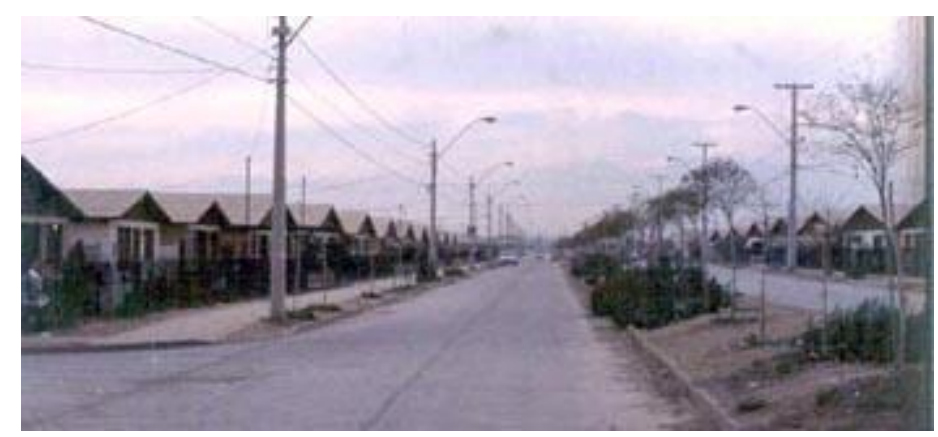

Figura No17: Loteo suburbano en Maipú, Santiago de Chile. Foto de C. Mawromatis P., 1996.

La reestructuración económica iniciada en Chile a mediados de los años 1970, junto a una progresiva tendencia a la concentración metropolitana, ha dado impulso a una intensificación del proceso de suburbanización de la ciudad de Santiago.

En materia de gestión urbana, los principios de liberalización y desregulación fueron formalmente incorporados en una modificación al Plan Regulador Intercomunal, en 1979, considerando al mercado como el factor determinante del desarrollo de las ciudades y sosteniendo que el concepto normativo de límite urbano era la causa del desequilibrio que conlleva la marcada diferencia entre valores del suelo urbano y rural. (de Mattos, 1999).

Surgió de esta manera una metrópolis segregada y fragmentada, en la cual aparecieron nuevas tipologías de edificaciones y espacios, tales como los shopping centers y malls comerciales, centros empresariales, centros de entretención, y conjuntos residenciales cerrados, entre otros, los que comienzan a incidir fuertemente en la estructura, morfología y movilidad de la Región Metropolitana de Santiago.

Paralelamente se ha generado una creciente demanda por infraestructura, la cual es aún bastante precaria en el caso de Santiago.

Si bien en 1985 se introdujeron importantes modificaciones al entonces Plan Regulador Intercomunal (1979), algunos de sus planteamientos básicos han permanecido vigentes hasta el día de hoy.

\section{Los balnearios en la costa central chilena}

Por otro lado, el suburbio disperso como modelo tipológico norteamericano, ha sido introducido previamente en Chile, en grandes proyectos inmobiliarios (segunda vivienda), en áreas contiguas a balnearios de la costa de la quinta región.

Tipológicamente la estructura vial y predial de estos proyectos, se presenta muy cercana al modelo norteamericano. Se conciben como islas de su entorno vernáculo, y atienden a un usuario de clase socioeconómica media-alta y alta, el cual depende del 
automóvil como principal medio de movilidad, y dispone mayoritariamente de más de un vehículo por núcleo familiar.

El primero de estos balnearios en Chile corresponde a Rocas de Santo Domingo (V Región).

Este balneario se inició en 1941 como una sociedad inmobiliaria, caracterizándose como balneario exclusivo, consolidándose como residencia permanente para muchos de sus propietarios y constituyéndose en uno de los más grandes proyectos inmobiliarios de Chile. La estructura urbana se desarrolla desde el acceso oriente y se prolonga en grandes avenidas radiales y concéntricas rodeando una colina y retornando hacia la costanera de la playa. Este balneario incluye un club de golf. (Munizaga, 1998):

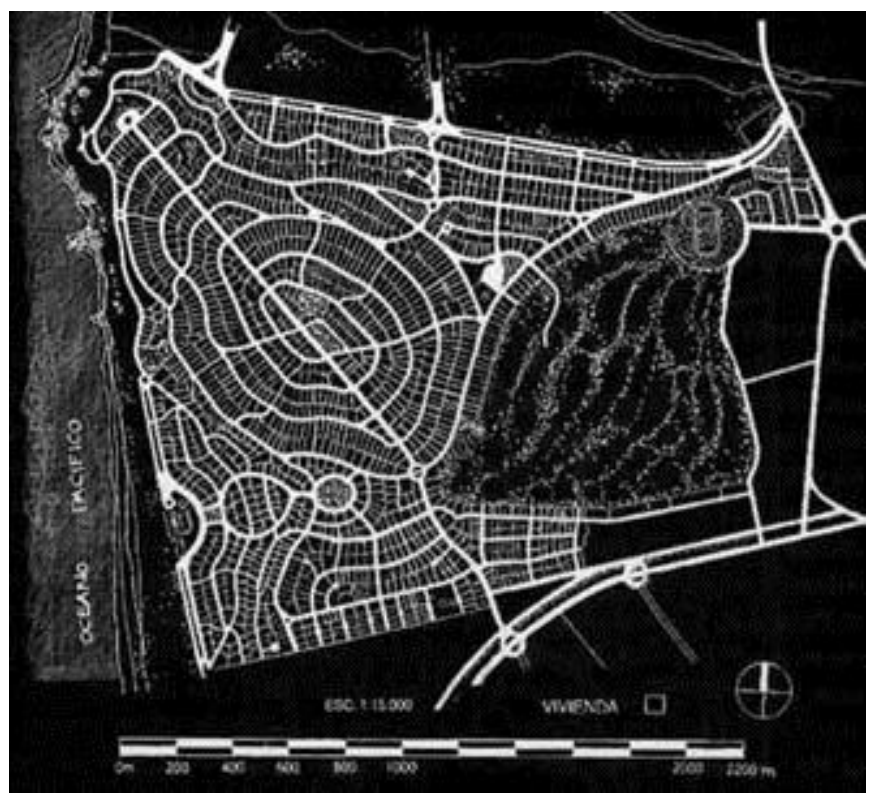

Figura No18: Balneario Rocas de Santo Domingo. V Región, Chile. Planta general.

Fuente; MUNIZAGA V., Gustavo, Macroarquitectura: Tipologías y Estrategias de Desarrollo Urbano. Textos Universitarios, Facultad de Arquitectura, Ediciones Universidad Católica de Chile, primera edición 1993, segunda edición ampliada y revisada 1999, p. 209.

Posteriormente, en una versión que representa más puramente el modelo norteamericano nos encontramos con el balneario de Marbella (Puchuncaví, V Región) el cual se estructura a partir de un sistema de colectores viales que van alimentando vías secundarias que finalmente rematan en cul de sacs. De esta manera se generan lotes o predios próximos que, sin embargo, se encuentran desvinculados unos de otros en lo referido a vialidad. Este desarrollo inmobiliario que está aún en realización, es 
privado, con viviendas unifamiliares y equipamientos compartidos, un complejo turístico y deportivo destinado a estratos socio económicos altos.

Desde su inicio en 1978, Marbella tiene una imagen fuerte de interioridad y unidad de diseño. El trazado inicial de un sistema troncal se desarrolló a partir de la vía interna de circunvalación desde la cual nacen los pasajes con cul de sacs, que distribuyen a agrupaciones residenciales de 7 a 15 lotes, bastante homogéneos. En su gran mayoría estos predios se ordenan alrededor de las canchas de golf. (Munizaga, 1998).

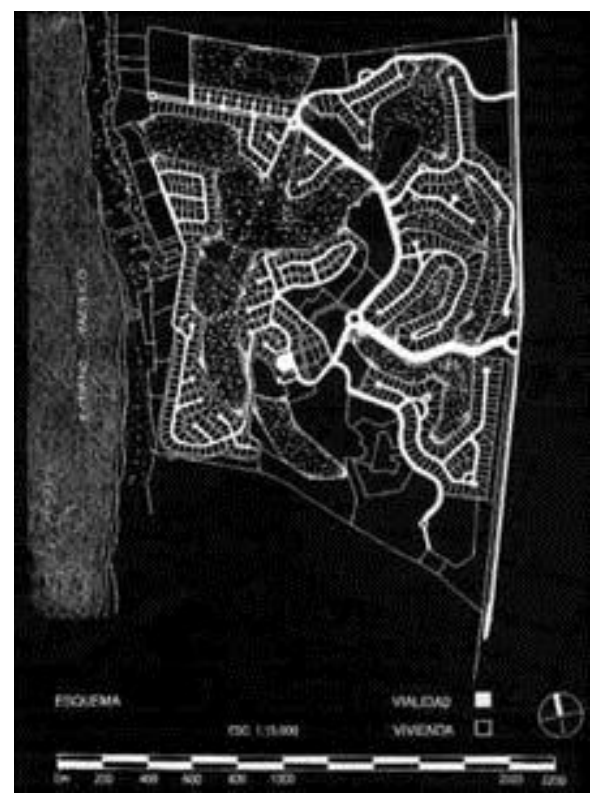

Figura N019: Marbella, V Región, Chile. Planta general, estructura vial.

Fuente; MUNIZAGA V., Gustavo, Macroarquitectura: Tipologías y Estrategias de Desarrollo Urbano. Textos Universitarios, Facultad de Arquitectura, Ediciones Universidad Católica de Chile, primera edición 1993, segunda edición ampliada y revisada 1999, p. 211.

Además de estos proyectos en los balnearios, han surgido en Chile los loteos -tipo parcelas de agrado- en los perímetros exteriores de los centros urbanos, los cuales están destinados a servir a una clientela de clase media alta y alta. Estas urbanizaciones cerradas, se organizan generalmente alrededor de canchas de golf, y presentan generalmente un acceso único y controlado.

Si bien en sus comienzos, estas subdivisiones se vendieron como propiedades de descanso insertas en paisajes naturales, hoy se presentan como única alternativa económicamente asequible para matrimonios jóvenes con hijos (de la clase media alta), que no poseen los medios para adquirir propiedades dentro del radio urbano de la metrópolis, y que requieren de una vivienda unifamiliar con jardín. 
En el mediano y largo plazo, la demanda por nueva infraestructura, la problemática de movilidad, y la pérdida de un ambiente natural y tranquilo, hará cada vez menos atractiva la vida en estos verdaderos "suburbios dispersos".

\section{¿Globalización o importación irreflexiva?}

En cuanto a la movilidad, se produce un fenómeno paradigmático de la importación irreflexiva de modelos urbanos, desatendiendo el marco global. Por un lado se adoptaron en la Región Metropolitana de Santiago, los instrumentos y las políticas de crecimiento que hicieron posible el surgimiento de un modelo de suburbio tipo "disperso norteamericano", favoreciendo una determinada postura de desarrollo económico. Por otro, se desestimaron las proyecciones que dicha decisión tendría en el mediano y largo plazo, por cuanto la expansión explosiva del límite urbano conlleva una demanda de infraestructura y servicios públicos que el país no es capaz de atender eficazmente. Además, se alteran los parámetros de movilidad, sin ofrecer una política coherente al respecto.

Mientras que en los EE.UU. la decisión política y económica de incrementar y privilegiar el aspecto comercial del desarrollo suburbano se tomó en conjunto con una política de desarrollo del sector automotriz, además de un apoyo decidido del sector público hacia la creación de una red de autopistas a nivel nacional, en Chile se adoptó meramente el aspecto comercial especulativo de la expansión urbana.

En efecto, a más de 20 años de la modificación de 1979 del Plan Regulador Intercomunal de Santiago 1960-1994, la demanda por el transporte público bordea el $69 \%$ de los viajes, contra el 31\% del transporte privado (SECTRA, 2000).

El problema se agrava si se toma en cuenta la descoordinación que existe entre los organismos de regulación del transporte público y los instrumentos vigentes que regulan la planificación urbana y territorial.

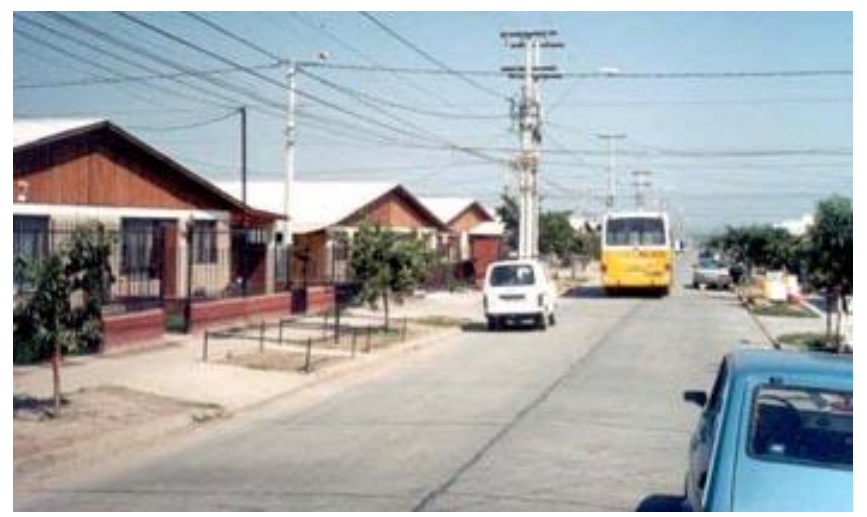

Figura N020: Transporte público y espacio urbano en la Comuna de Maipú, Santiago. Suburbanización de la Metrópolis.

Foto de C. Mawromatis P., 1997. 
A pesar de que en los últimos años se ha notado -al menos en el discurso oficial- una mayor sensibilidad en cuanto a favorecer la creación de barrios y fomentar los usos mixtos en las urbanizaciones de subsidio estatal, en la práctica los instrumentos de regulación vigentes permiten la proliferación del modelo del suburbio disperso del tipo norteamericano.

Por otro lado, los postulados del Nuevo Urbanismo son escasamente conocidos dentro del ámbito académico y menos en el medio de los promotores inmobiliarios, por lo cual aún no es posible concretar algún proyecto que integre la diversidad que ofrecen estos ejemplos en los EE.UU.

Si bien la realidad histórica y cultural de los EE.UU. es distinta en su origen a la chilena, el fenómeno de la globalización y la dependencia cultural que tiene Chile con los EE.UU., permitiría imaginar una aceleración de la adopción del Nuevo Urbanismo como alternativa al suburbio disperso, como una opción, siempre que el éxito comercial se encontrara probado y consolidado previamente en su lugar de origen.

\section{Referencias}

\section{Bibliografía}

CALTHORPE, Peter, 1993. The Next American Metropolis, Ecology, Community, and the American Dream. Princeton Architectural Press.

CÁRDENAS J., Luz Alicia, y MAWROMATIS P., Constantino, 1999. "Urban form at the fringe of metropolitan Santiago. A result of a normative or profitability plan?". En Revista de Urbanismo $N^{\circ} 1$, revista digital, Departamento de Urbanismo, F.A.U. de la Universidad de Chile, diciembre de 1999, I.S.S.N. 0717-5051, [en línea] http://revistaurbanismo.uchile.cl

CONGRESS FOR THE NEW URBANISM, 2000. Charter of the New Urbanism. McGrawHill, New York.

DE MATTOS, Carlos A., 1999. "Globalización y Metropolización". En: Revista Latinoamericana de Estudios Urbano Regionales, EURE, N76, Vol. XXV, diciembre, Instituto de Estudios Urbanos, Pontificia Universidad Católica de Chile.

DUANY, Andres; PLATER-ZYBERK, Elizabeth, 1991. Towns and Townmaking Principles. Harvard University Graduate School of Design. Rizzoli, New York.

EASTERLING, Keller, 1993. American Townplans, a comparative time line. Princeton Architectural Press.

KATZ, Peter, 1994. The New Urbanism. Toward an Architecture of Community. Ed. Mc Graw-Hill, Inc. 
KOSTOF, Spiro, 1991; The City Shaped, Urban Patterns and Meanings Through History. Thames and Hudson Ltd., London.

KOSTOF, Spiro, 1992. The City Assembled, The Elements of Urban Form Through History. Thames and Hudson Ltd., London.

ARCHITECTURAL DESIGN PROFILE, 1984. Ed. Dr. Andreas Papadakis. Ed Invitado, Demetri Porphyrios. Leon Krier. Homes, Palaces, Cities. Gran Bretaña, 1984.

MAWROMATIS PAZDERKA, Constantino, 1995. "La cultura del suburbio y las nuevas alternativas de diseño urbano en EE.UU.". En revista De Arquitectura No6, F.A.U. de la Universidad de Chile, septiembre de 1995, I.S.S.N. 0716-8772, pp.8-9 [resúmenes, en línea] www.uchile.cl/facultades/arquitectura/publicaciones/revista.html

MAWROMATIS P., Constantino, 2000. "El divorcio entre los instrumentos de planificación y la morfología urbana en el perímetro metropolitano de Santiago de Chile: el caso de Maipú". En Revista de Urbanismo $N^{\circ} 2$, revista digital, Departamento de Urbanismo, F.A.U. de la Universidad de Chile, marzo de 2000, I.S.S.N. 0717-5051 [en línea] http://revistaurbanismo.uchile.cl

MUNIZAGA VIGIL, Gustavo, 1998. Macroarquitectura: Tipologías y Estrategias de Desarrollo Urbano. Textos Universitarios, Facultad de Arquitectura, Ediciones Universidad Católica de Chile.

POZUETA, Julio, 2001. Movilidad y Planeamiento Sostenible: Hacia una consideración inteligente del transporte y la movilidad en el planeamiento y en el diseño urbano, julio de 2001.

POZUETA, Julio, SÁNCHEZ-FAYOS, Teresa, VILLACAÑAS, Silvia, 1998. La regulación de la dotación de plazas de estacionamiento en el marco de la congestión. Cuadernos de Investigación urbanística, N07, diciembre de 1998.

SECTRA, Gobierno de Chile, 2002. Resumen Ejecutivo del Plan de Transporte Urbano de Santiago, 2000-2006; 26 de octubre de 2000.

\section{Notas}

(1) Los proyectos inmobiliarios acogidos al FHA (Federal Housing Administration) se rigen bajo una serie de recomendaciones que apuntan a un modelo suburbano orientado a minimizar los riesgos de créditos hipotecarios y preferenciar el desarrollo inmobiliario del sector privado. Se prevalecen en la estructura de estos proyectos consideraciones de tipo económicas como: accesos mínimos, manzanas elongadas, vialidad enfocada al vehículo privado, el uso del cul-de-sac, la maximización de predios privados hacia un frente público mínimo, estandarización predial, y la total ausencia de diversidad de usos del suelo.

(2) Cifra citada por Duany y Plater-Zyberk, 1991, Op.Cit.). 


\section{REVISTA DE \\ URBANISMO \\ ISSN 0717-5051}

http://revistaurbanismo.uchile.cl
Movilidad en los suburbios dispersos y el Nuevo Urbanismo en los Estados Unidos de América: ¿Importación irreflexiva desde Chile?

N. de R.: Traducciones desde el inglés, de C. Mawromatis P. Las ilustraciones que se presenta, se reproducen sin fines de lucro, con objetivos estrictamente culturales. Este escrito corresponde a materias relacionadas con el proyecto de Tesis Doctoral de C.M.P. 